\title{
Machine learning in predictive toxicology: recent applications and future directions for classification models
}

Marcus W. H. Wang, Jonathan M. Goodman, Timothy E. H. Allen

\section{Supporting Information}

\section{Table of Contents}

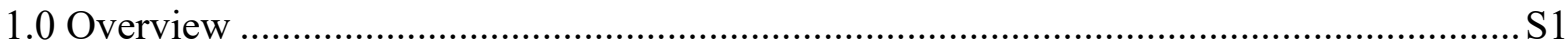

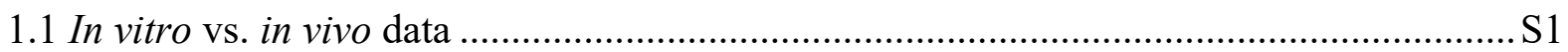

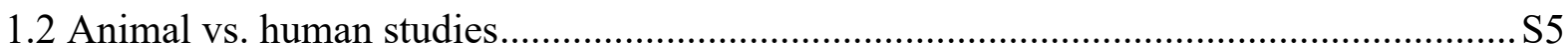

\subsection{Overview}

This document contains the data used to plot in vitro vs. in vivo, as well as animal vs. human charts based on the data in Table S1 and Table S3 respectively. This gives an idea whether the model performance depends on any of these factors.

\subsection{In vitro vs. in vivo data}

The in vitro and in vivo plots were generated based on the data in Table S1. Entries where the data used (in vitro/in vivo) is unclear were omitted during the plot. Accuracy was chosen to represent the model performance. In the event that the paper does not have an accuracy value, AUC was used instead. The accuracy/AUC (\%) used for plotting Figure S1 and S2 are tabulated in a column in Table S1. In vitro and in vivo data were assigned $\mathrm{x}$-axis values of 0.5 and 1 respectively in order to differentiate them on the same plot. This is also recorded in Table S1.

Figure S1 shows no clear distinction between in vivo and in vitro data, other than the larger variation between the accuracy/AUC(\%) for in vivo studies as compared to in vitro studies. For Figure S2, there is a lack of data for in vitro cardiotoxicity and in vivo hepatotoxicity that was obtained using the search protocol in the main paper. For all endpoints, the in vitro data has a higher mean accuracy/AUC(\%) than the in vivo data. This is expected as in vitro experiments can be controlled more precisely. 
Table S1: Data used for plotting Figures S1 and S2.

\begin{tabular}{|c|c|c|c|c|c|c|c|c|c|}
\hline No. & $\begin{array}{l}\text { Machine } \\
\text { learning } \\
\text { method }\end{array}$ & $\begin{array}{l}\text { Toxicity } \\
\text { endpoint }\end{array}$ & $\begin{array}{c}\text { Accuracy } \\
(\%)\end{array}$ & $\begin{array}{c}\text { AUC } \\
(\%)\end{array}$ & Q/AUC & $\begin{array}{l}\text { Dataset } \\
\text { size }\end{array}$ & $\begin{array}{c}\text { In vitro } \\
(0.5) / \\
\text { In vivo } \\
(1) \\
\end{array}$ & Comments & $\begin{array}{c}\text { Reference } \\
\text { Number }\end{array}$ \\
\hline 1 & $\begin{array}{c}\text { Regression } \\
\text { (Linear) }\end{array}$ & Cardiotoxicity & - & 75 & 75 & 1917 & 1 & & 159 \\
\hline 2 & $\begin{array}{l}\text { Regression } \\
\text { (Ridge) }\end{array}$ & Cardiotoxicity & - & 77 & 77 & 1917 & 1 & & 159 \\
\hline 3 & $\begin{array}{c}\text { Regression } \\
\text { (Partial } \\
\text { least } \\
\text { square) }\end{array}$ & $\begin{array}{l}\text { Molecular } \\
\text { toxicity }\end{array}$ & 82 & - & 82 & 2849 & 0.5 & & 170 \\
\hline 4 & $\mathrm{kNN}$ & $\begin{array}{l}\text { Aquatic } \\
\text { toxicity }\end{array}$ & 84 & 92 & 84 & 1005 & 0.5 & & 174 \\
\hline 5 & $\mathrm{kNN}$ & Cardiotoxicity & 82 & 78 & 82 & 206 & 1 & & 176 \\
\hline 6 & $\mathrm{kNN}$ & Genotoxicity & 86 & 93 & 86 & 576 & 0.5 & & 177 \\
\hline 7 & $\mathrm{kNN}$ & Hepatotoxicity & 62 & 52 & 62 & 978 & - & $\begin{array}{c}\text { Collection of } \\
\text { studies (unclear) }\end{array}$ & 178 \\
\hline 8 & $\mathrm{kNN}$ & Hepatotoxicity & 78 & - & 78 & 1274 & - & $\begin{array}{c}\text { Collection of } \\
\text { studies (unclear) }\end{array}$ & 139 \\
\hline 9 & DT & Genotoxicity & 82 & 81 & 82 & 576 & 0.5 & & 177 \\
\hline 10 & NB & $\begin{array}{l}\text { Aquatic } \\
\text { toxicity }\end{array}$ & 77 & 81 & 77 & 1005 & 0.5 & & 174 \\
\hline 11 & NB & Carcinogenicity & $68 \pm 2$ & - & 68 & 834 & 0.5 & & 142 \\
\hline 12 & NB & $\begin{array}{l}\text { Developmental } \\
\text { toxicity }\end{array}$ & 83 & - & 83 & 232 & 0.5 & & 143 \\
\hline 13 & NB & Genotoxicity & 85 & 90 & 85 & 576 & 0.5 & $\begin{array}{c}* \text { in vivo } \\
\text { micronucleus } \\
\text { assay }\end{array}$ & 177 \\
\hline 14 & NB & Immunotoxicity & - & 78 & 78 & 44615 & 1 & & 145 \\
\hline 15 & NB & $\begin{array}{l}\text { Mitochondrial } \\
\text { toxicity }\end{array}$ & $81 \pm 1$ & - & 81 & 226 & 1 & & 148 \\
\hline 16 & NB & Mutagenicity & $90.9 \pm 0.3$ & - & 91 & 5159 & 1 & & 146 \\
\hline 17 & NB & Mutagenicity & 65 & - & 65 & 3903 & 1 & & 147 \\
\hline 18 & NB & Myelotoxicity & $82 \pm 3$ & - & 82 & 727 & 0.5 & & 149 \\
\hline 19 & NB & $\begin{array}{c}\text { Respiratory } \\
\text { toxicity }\end{array}$ & 84 & - & 84 & 993 & 0.5 & & 151 \\
\hline 20 & NB & $\begin{array}{c}\text { Urinary tract } \\
\text { toxicity }\end{array}$ & 84 & - & 84 & 173 & 0.5 & & 152 \\
\hline 21 & SVM & $\begin{array}{c}\text { Acute oral } \\
\text { toxicity }\end{array}$ & 90 & - & 90 & 8102 & 0.5 & & 46 \\
\hline 22 & SVM & Acute toxicity & 70 & 72 & 70 & 321 & 0.5 & & 153 \\
\hline 23 & SVM & $\begin{array}{l}\text { Aquatic } \\
\text { toxicity }\end{array}$ & 89 & 94 & 89 & 1005 & 0.5 & & 174 \\
\hline 24 & SVM & Carcinogenicity & $68 \pm 3$ & $\begin{array}{c}73 \pm \\
3\end{array}$ & 68 & 802 & 0.5 & & 154 \\
\hline 25 & SVM & Carcinogenicity & 78 & - & 78 & 661 & 0.5 & & 175 \\
\hline 26 & SVM & Cardiotoxicity & 86 & 72 & 86 & 206 & 1 & & 176 \\
\hline 27 & SVM & Cardiotoxicity & 87 & - & 87 & 1501 & 1 & & 155 \\
\hline 28 & SVM & Genotoxicity & 89 & 95 & 89 & 576 & 0.5 & & 177 \\
\hline
\end{tabular}




\begin{tabular}{|c|c|c|c|c|c|c|c|c|c|}
\hline 29 & SVM & Hepatotoxicity & 75 & 61 & 75 & 978 & - & $\begin{array}{c}\text { Collection of } \\
\text { studies (unclear) }\end{array}$ & 178 \\
\hline 30 & SVM & Hepatotoxicity & 83 & 89 & 83 & 1731 & 0.5 & & 156 \\
\hline 31 & SVM & Mutagenicity & 72 & - & 72 & 1696 & 1 & & 157 \\
\hline 32 & SVM & Ototoxicity & 85 & - & 85 & 536 & 0.5 & & 158 \\
\hline 33 & $\begin{array}{l}\text { Ensemble } \\
\text { learning/RF }\end{array}$ & $\begin{array}{l}\text { Aquatic } \\
\text { toxicity }\end{array}$ & 86 & 93 & 86 & 1005 & 0.5 & & 174 \\
\hline 34 & $\begin{array}{l}\text { Ensemble } \\
\text { learning/RF }\end{array}$ & Carcinogenicity & $70 \pm 3$ & $\begin{array}{c}77 \pm \\
3\end{array}$ & 70 & 802 & 0.5 & & 154 \\
\hline 35 & $\begin{array}{l}\text { Ensemble } \\
\text { learning/RF }\end{array}$ & Carcinogenicity & 74 & - & 74 & 661 & 0.5 & & 175 \\
\hline 36 & $\begin{array}{l}\text { Ensemble } \\
\text { learning/RF }\end{array}$ & Carcinogenicity & $68 \pm 3$ & $\begin{array}{c}74 \pm \\
3\end{array}$ & 68 & 802 & 0.5 & & 154 \\
\hline 37 & $\begin{array}{l}\text { Ensemble } \\
\text { learning/RF }\end{array}$ & Cardiotoxicity & 82 & 94 & 82 & 2901 & 1 & & 160 \\
\hline 38 & $\begin{array}{l}\text { Ensemble } \\
\text { learning/RF }\end{array}$ & Cardiotoxicity & 97 & - & 97 & 522 & 1 & & 161 \\
\hline 39 & $\begin{array}{l}\text { Ensemble } \\
\text { learning/RF }\end{array}$ & Genotoxicity & 94 & 96 & 94 & 576 & 0.5 & & 177 \\
\hline 40 & $\begin{array}{l}\text { Ensemble } \\
\text { learning/RF }\end{array}$ & Hepatotoxicity & $69 \pm 3$ & $\begin{array}{c}75 \pm \\
3\end{array}$ & 69 & 993 & 1 & & 163 \\
\hline 41 & $\begin{array}{l}\text { Ensemble } \\
\text { learning/RF }\end{array}$ & Hepatotoxicity & 74 & 79 & 74 & 281 & 0.5 & & 162 \\
\hline 42 & $\begin{array}{l}\text { Ensemble } \\
\text { learning/RF }\end{array}$ & Hepatotoxicity & $71 \pm 3$ & $\begin{array}{c}76 \pm \\
2\end{array}$ & 71 & 1117 & 0.5 & & 163 \\
\hline 43 & $\begin{array}{l}\text { Ensemble } \\
\text { learning/RF }\end{array}$ & Hepatotoxicity & 73 & - & 73 & 1274 & - & $\begin{array}{l}\text { Collection of } \\
\text { studies (unclear) }\end{array}$ & 139 \\
\hline 44 & NN (ANN) & Acute toxicity & - & 70 & 70 & 321 & 0.5 & & 153 \\
\hline 45 & NN (ANN) & $\begin{array}{l}\text { Aquatic } \\
\text { toxicity }\end{array}$ & 88 & 94 & 88 & 1005 & 0.5 & & 174 \\
\hline 46 & NN (ANN) & Genotoxicity & 87 & 94 & 87 & 576 & 0.5 & & 177 \\
\hline 47 & NN (ANN) & Hepatotoxicity & 82 & - & 82 & 475 & 0.5 & & 166 \\
\hline 48 & NN (ANN) & Mutagenicity & 60 & - & 60 & 1696 & 1 & & 157 \\
\hline 49 & NN (ANN) & Mutagenicity & 80 & 87 & 80 & 6094 & 1 & & 167 \\
\hline 50 & NN (DNN) & Cardiotoxicity & 93 & 97 & 93 & 3954 & 1 & & 165 \\
\hline 51 & NN (DNN) & Cardiotoxicity & 98 & - & 98 & 522 & 1 & & 161 \\
\hline 52 & NN (DNN) & General & - & 84 & 84 & 11764 & 1 & $\begin{array}{c}\text { * Should be } \\
\text { animal studies, but } \\
\text { human relevant }\end{array}$ & 17 \\
\hline 53 & NN (DNN) & Hepatotoxicity & 81 & - & 81 & 475 & 0.5 & & 166 \\
\hline 54 & $\begin{array}{l}\text { NN (Graph } \\
\text { CNN) }\end{array}$ & Cardiotoxicity & - & 96 & 96 & 3954 & 0.5 & & 165 \\
\hline 55 & NN (CNN) & General & - & 78 & 78 & 10588 & 1 & $\begin{array}{l}\text { * Should be } \\
\text { animal studies, but } \\
\text { human relevant }\end{array}$ & 168 \\
\hline 56 & NN (CNN) & General & - & 85 & 85 & 7438 & 1 & $\begin{array}{c}\text { * Should be } \\
\text { animal studies, but } \\
\text { human relevant }\end{array}$ & 169 \\
\hline 57 & NN (CNN) & Hepatotoxicity & 63 & 62 & 63 & 7630 & - & $\begin{array}{l}\text { Collection of } \\
\text { studies (unclear) }\end{array}$ & 171 \\
\hline
\end{tabular}

Figure S1: Overall in vitro vs. in vivo plot based on Table S1 


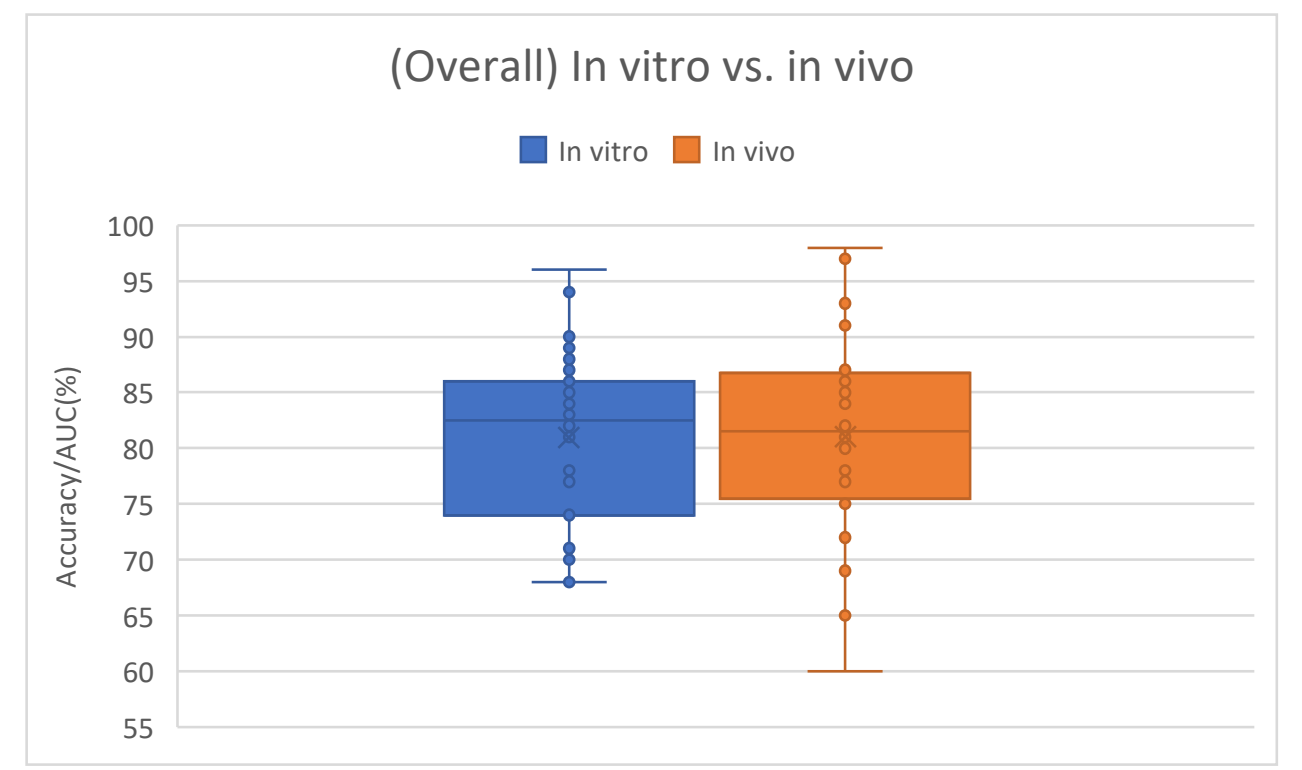

Figure S2: In vitro vs. in vivo plot based on Table S1, separated by endpoints

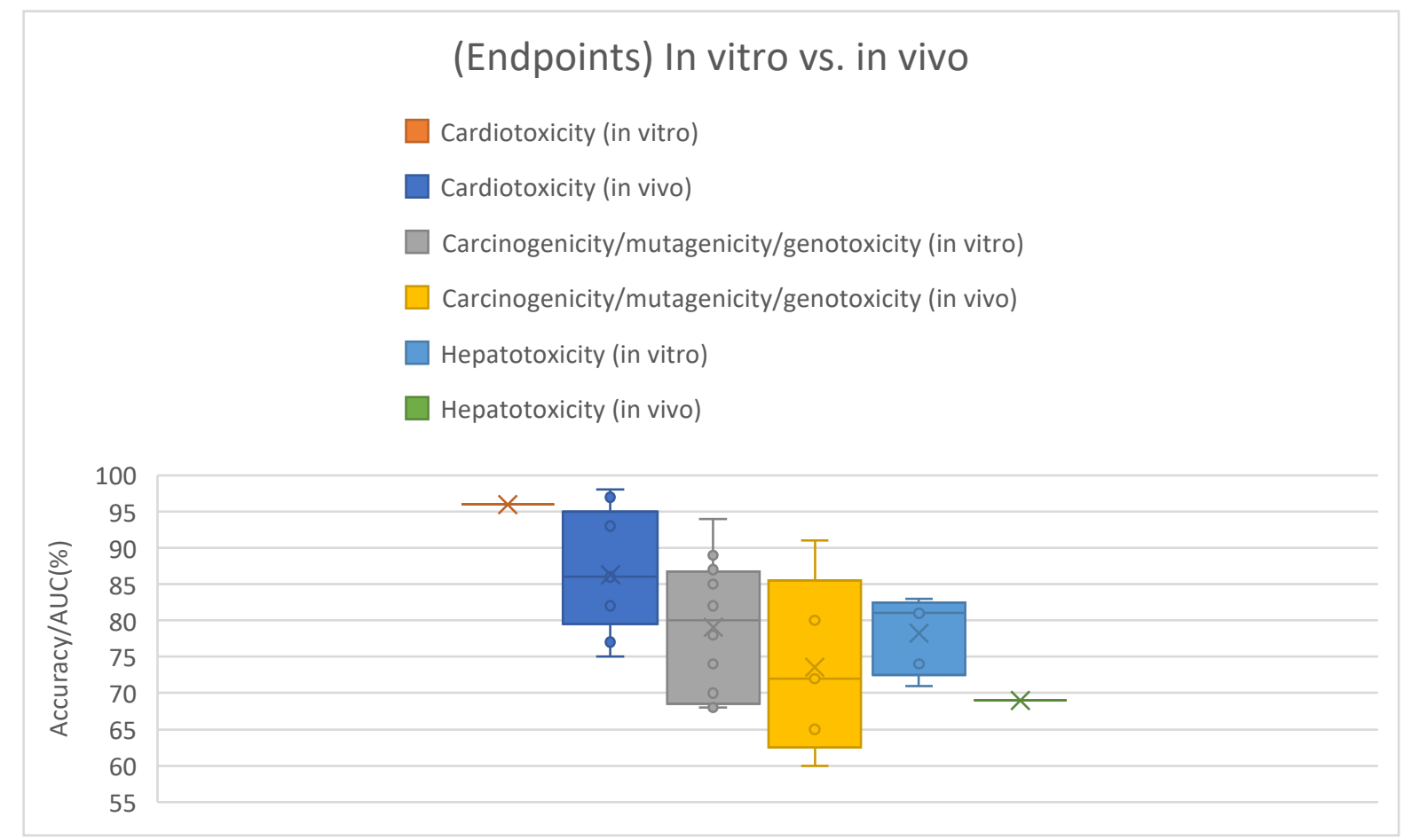




\subsection{Animal vs. human studies}

The animal vs. human plots were generated based on the data in Table S3. Entries where the data used (animal vs. human) is unclear were omitted during the plot. Accuracy was chosen to represent the model performance. In the event that the paper does not have an accuracy value, AUC was used instead. The accuracy/AUC (\%) used for plotting Figure S3 and S4 are tabulated in a column in Table S3. Data from animal and human studies were assigned x-axis values of 0.5 and 1 respectively in order to differentiate them on the same plot. This is also recorded in Table S3.

Figure S3 shows no clear distinction between animal vs. human data, other than the smaller variation between the accuracy/AUC $(\%)$ for human studies as compared to animal studies. For Figure S4, there is no animal data for hepatotoxicity that was obtained using the search protocol in the main paper. For cardiotoxicity, the mean accuracy/AUC $(\%)$ for animal studies is larger than the mean accuracy/AUC $(\%)$ for human studies. However, this trend is reversed for carcinogenicity/mutagenicity/genotoxicity. 
Table S2: Data used for plotting Figures S3 and S4.

\begin{tabular}{|c|c|c|c|c|c|c|c|c|c|}
\hline No. & $\begin{array}{l}\text { Machine } \\
\text { learning } \\
\text { method }\end{array}$ & $\begin{array}{c}\text { Toxicity } \\
\text { endpoint }\end{array}$ & $\begin{array}{c}\text { Accuracy } \\
(\%)\end{array}$ & $\begin{array}{c}\text { AUC } \\
(\%)\end{array}$ & Q/AUC & $\begin{array}{l}\text { Dataset } \\
\text { size }\end{array}$ & $\begin{array}{c}\text { Animal } \\
(0.5) / \\
\text { Human } \\
(1)\end{array}$ & Comments & $\begin{array}{c}\text { Reference } \\
\text { Number }\end{array}$ \\
\hline 1 & $\begin{array}{l}\text { Regression } \\
\text { (Linear) }\end{array}$ & Cardiotoxicity & - & 75 & 75 & 1917 & 1 & & 159 \\
\hline 2 & $\begin{array}{l}\text { Regression } \\
\text { (Ridge) }\end{array}$ & Cardiotoxicity & - & 77 & 77 & 1917 & 1 & & 159 \\
\hline 3 & $\begin{array}{c}\text { Regression } \\
\text { (Partial } \\
\text { least } \\
\text { square) }\end{array}$ & $\begin{array}{l}\text { Molecular } \\
\text { toxicity }\end{array}$ & 82 & - & 82 & 2849 & 0.5 & & 170 \\
\hline 4 & $\mathrm{kNN}$ & $\begin{array}{l}\text { Aquatic } \\
\text { toxicity }\end{array}$ & 84 & 92 & 84 & 1005 & 0.5 & & 174 \\
\hline 5 & $\mathrm{kNN}$ & Cardiotoxicity & 82 & 78 & 82 & 206 & 1 & & 176 \\
\hline 6 & kNN & Genotoxicity & 86 & 93 & 86 & 576 & 1 & & 177 \\
\hline 7 & $\mathrm{kNN}$ & Hepatotoxicity & 62 & 52 & 62 & 978 & - & $\begin{array}{c}\text { Collection of } \\
\text { studies (unclear) }\end{array}$ & 178 \\
\hline 8 & $\mathrm{kNN}$ & Hepatotoxicity & 78 & - & 78 & 1274 & - & $\begin{array}{c}\text { Collection of } \\
\text { studies (unclear) }\end{array}$ & 139 \\
\hline 9 & DT & Genotoxicity & 82 & 81 & 82 & 576 & 1 & & 177 \\
\hline 10 & NB & $\begin{array}{l}\text { Aquatic } \\
\text { toxicity }\end{array}$ & 77 & 81 & 77 & 1005 & 0.5 & & 174 \\
\hline 11 & NB & Carcinogenicity & $68 \pm 2$ & - & 68 & 834 & 0.5 & & 142 \\
\hline 12 & NB & $\begin{array}{l}\text { Developmental } \\
\text { toxicity }\end{array}$ & 83 & - & 83 & 232 & 0.5 & & 143 \\
\hline 13 & NB & Genotoxicity & 85 & 90 & 85 & 576 & 1 & $\begin{array}{c}* \text { in vivo } \\
\text { micronucleus assay }\end{array}$ & 177 \\
\hline 14 & NB & Immunotoxicity & - & 78 & 78 & 44615 & 1 & & 145 \\
\hline 15 & NB & Mutagenicity & $90.9 \pm 0.3$ & - & 91 & 5159 & 0.5 & & 148 \\
\hline 16 & NB & Mutagenicity & 65 & - & 65 & 3903 & 0.5 & & 146 \\
\hline 17 & NB & $\begin{array}{l}\text { Mitochondrial } \\
\text { toxicity }\end{array}$ & $81 \pm 1$ & - & 81 & 226 & 1 & & 147 \\
\hline 18 & NB & Myelotoxicity & $82 \pm 3$ & - & 82 & 727 & 1 & & 149 \\
\hline 19 & NB & $\begin{array}{c}\text { Respiratory } \\
\text { toxicity }\end{array}$ & 84 & - & 84 & 993 & 1 & & 151 \\
\hline 20 & NB & $\begin{array}{l}\text { Urinary tract } \\
\text { toxicity }\end{array}$ & 84 & - & 84 & 173 & 0.5 & & 152 \\
\hline 21 & SVM & Acute toxicity & 70 & 72 & 70 & 321 & 1 & & 46 \\
\hline 22 & SVM & $\begin{array}{c}\text { Acute oral } \\
\text { toxicity }\end{array}$ & 90 & - & 90 & 8102 & 0.5 & & 153 \\
\hline 23 & SVM & $\begin{array}{l}\text { Aquatic } \\
\text { toxicity }\end{array}$ & 89 & 94 & 89 & 1005 & 0.5 & & 174 \\
\hline 24 & SVM & Carcinogenicity & $68 \pm 3$ & $\begin{array}{c}73 \pm \\
3\end{array}$ & 68 & 802 & 0.5 & & 154 \\
\hline 25 & SVM & Carcinogenicity & 78 & - & 78 & 661 & 0.5 & & 175 \\
\hline 26 & SVM & Cardiotoxicity & 86 & 72 & 86 & 206 & 1 & & 176 \\
\hline 27 & SVM & Cardiotoxicity & 87 & - & 87 & 1501 & 1 & & 155 \\
\hline 28 & SVM & Genotoxicity & 89 & 95 & 89 & 576 & 1 & & 177 \\
\hline
\end{tabular}




\begin{tabular}{|c|c|c|c|c|c|c|c|c|c|}
\hline 29 & SVM & Hepatotoxicity & 75 & 61 & 75 & 978 & - & $\begin{array}{c}\text { Collection of } \\
\text { studies (unclear) }\end{array}$ & 178 \\
\hline 30 & SVM & Hepatotoxicity & 83 & 89 & 83 & 1731 & 1 & & 156 \\
\hline 31 & SVM & Mutagenicity & 72 & - & 72 & 1696 & 0.5 & & 157 \\
\hline 32 & SVM & Ototoxicity & 85 & - & 85 & 536 & 1 & & 158 \\
\hline 33 & $\begin{array}{c}\text { Ensemble } \\
\text { learning/RF }\end{array}$ & $\begin{array}{l}\text { Aquatic } \\
\text { toxicity }\end{array}$ & 86 & 93 & 86 & 1005 & 0.5 & & 174 \\
\hline 34 & $\begin{array}{c}\text { Ensemble } \\
\text { learning/RF }\end{array}$ & Carcinogenicity & 74 & - & 74 & 661 & 0.5 & & 154 \\
\hline 35 & $\begin{array}{c}\text { Ensemble } \\
\text { learning/RF }\end{array}$ & Carcinogenicity & $68 \pm 3$ & $\begin{array}{c}74 \pm \\
3\end{array}$ & 68 & 802 & 0.5 & & 175 \\
\hline 36 & $\begin{array}{c}\text { Ensemble } \\
\text { learning/RF }\end{array}$ & Carcinogenicity & $70 \pm 3$ & $\begin{array}{c}77 \pm \\
3\end{array}$ & 70 & 802 & 0.5 & & 154 \\
\hline 37 & $\begin{array}{c}\text { Ensemble } \\
\text { learning/RF }\end{array}$ & Cardiotoxicity & 82 & 94 & 82 & 2901 & 0.5 & & 160 \\
\hline 38 & $\begin{array}{c}\text { Ensemble } \\
\text { learning/RF }\end{array}$ & Cardiotoxicity & 97 & - & 97 & 522 & 0.5 & & 161 \\
\hline 39 & $\begin{array}{c}\text { Ensemble } \\
\text { learning/RF }\end{array}$ & Genotoxicity & 94 & 96 & 94 & 576 & 1 & & 177 \\
\hline 40 & $\begin{array}{c}\text { Ensemble } \\
\text { learning/RF }\end{array}$ & Hepatotoxicity & $69 \pm 3$ & $\begin{array}{c}75 \pm \\
3\end{array}$ & 69 & 993 & 1 & & 163 \\
\hline 41 & $\begin{array}{c}\text { Ensemble } \\
\text { learning/RF }\end{array}$ & Hepatotoxicity & 74 & 79 & 74 & 281 & 1 & & 162 \\
\hline 42 & $\begin{array}{c}\text { Ensemble } \\
\text { learning/RF }\end{array}$ & Hepatotoxicity & $71 \pm 3$ & $\begin{array}{c}76 \pm \\
2\end{array}$ & 71 & 1117 & 1 & & 163 \\
\hline 43 & $\begin{array}{c}\text { Ensemble } \\
\text { learning/RF }\end{array}$ & Hepatotoxicity & 73 & - & 73 & 1274 & - & $\begin{array}{c}\text { Collection of } \\
\text { studies (unclear) }\end{array}$ & 139 \\
\hline 44 & $\mathrm{NN}(\mathrm{ANN})$ & Acute toxicity & - & 70 & 70 & 321 & 1 & & 153 \\
\hline 45 & NN (ANN) & $\begin{array}{l}\text { Aquatic } \\
\text { toxicity }\end{array}$ & 88 & 94 & 88 & 1005 & 0.5 & & 174 \\
\hline 46 & NN (ANN) & Genotoxicity & 87 & 94 & 87 & 576 & 1 & & 177 \\
\hline 47 & NN (ANN) & Hepatotoxicity & 82 & - & 82 & 475 & 1 & & 166 \\
\hline 48 & $\mathrm{NN}(\mathrm{ANN})$ & Mutagenicity & 60 & - & 60 & 1696 & 0.5 & & 157 \\
\hline 49 & $\mathrm{NN}(\mathrm{ANN})$ & Mutagenicity & 80 & 87 & 80 & 6094 & 0.5 & & 167 \\
\hline 50 & NN (DNN) & Cardiotoxicity & 93 & 97 & 93 & 3954 & 1 & & 165 \\
\hline 51 & NN (DNN) & Cardiotoxicity & 98 & - & 98 & 522 & 0.5 & & 161 \\
\hline 52 & $\mathrm{NN}(\mathrm{DNN})$ & General & - & 84 & 84 & 11764 & 0.5 & $\begin{array}{c}\text { * Should be animal } \\
\text { studies, but human } \\
\text { relevant }\end{array}$ & 17 \\
\hline 53 & NN (DNN) & Hepatotoxicity & 81 & - & 81 & 475 & 1 & & 166 \\
\hline 54 & $\begin{array}{l}\text { NN (Graph } \\
\text { CNN) }\end{array}$ & Cardiotoxicity & - & 96 & 96 & 3954 & 1 & & 165 \\
\hline 55 & $\mathrm{NN}(\mathrm{CNN})$ & General & - & 78 & 78 & 10588 & 0.5 & $\begin{array}{c}\text { * Should be animal } \\
\text { studies, but human } \\
\text { relevant }\end{array}$ & 168 \\
\hline 56 & $\mathrm{NN}(\mathrm{CNN})$ & General & - & 85 & 85 & 7438 & 0.5 & $\begin{array}{c}\text { * Should be animal } \\
\text { studies, but human } \\
\text { relevant }\end{array}$ & 169 \\
\hline 57 & $\mathrm{NN}(\mathrm{CNN})$ & Hepatotoxicity & 63 & 62 & 63 & 7630 & - & $\begin{array}{c}\text { Collection of } \\
\text { studies (unclear) }\end{array}$ & 171 \\
\hline
\end{tabular}


Figure S3: Overall animal vs. human plot based on Table S2

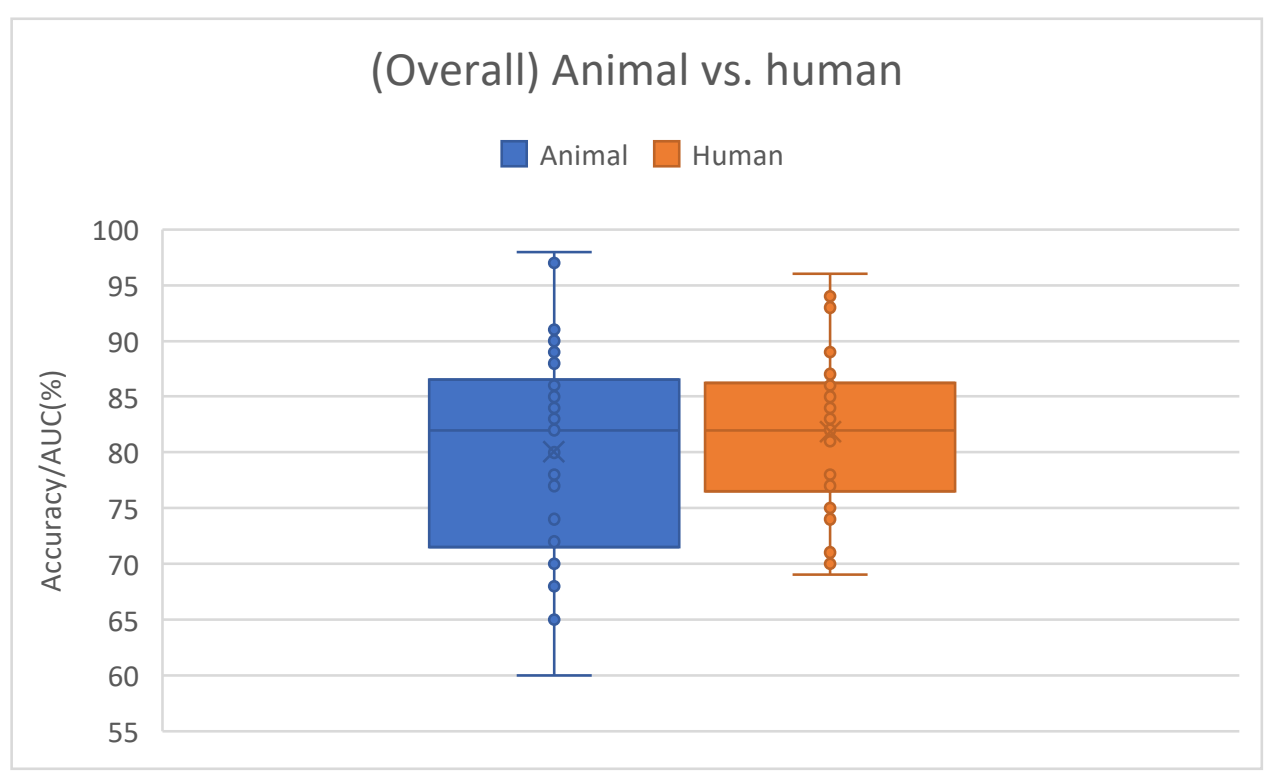

Figure S4: Animal vs. human plot based on Table S2, separated by endpoints

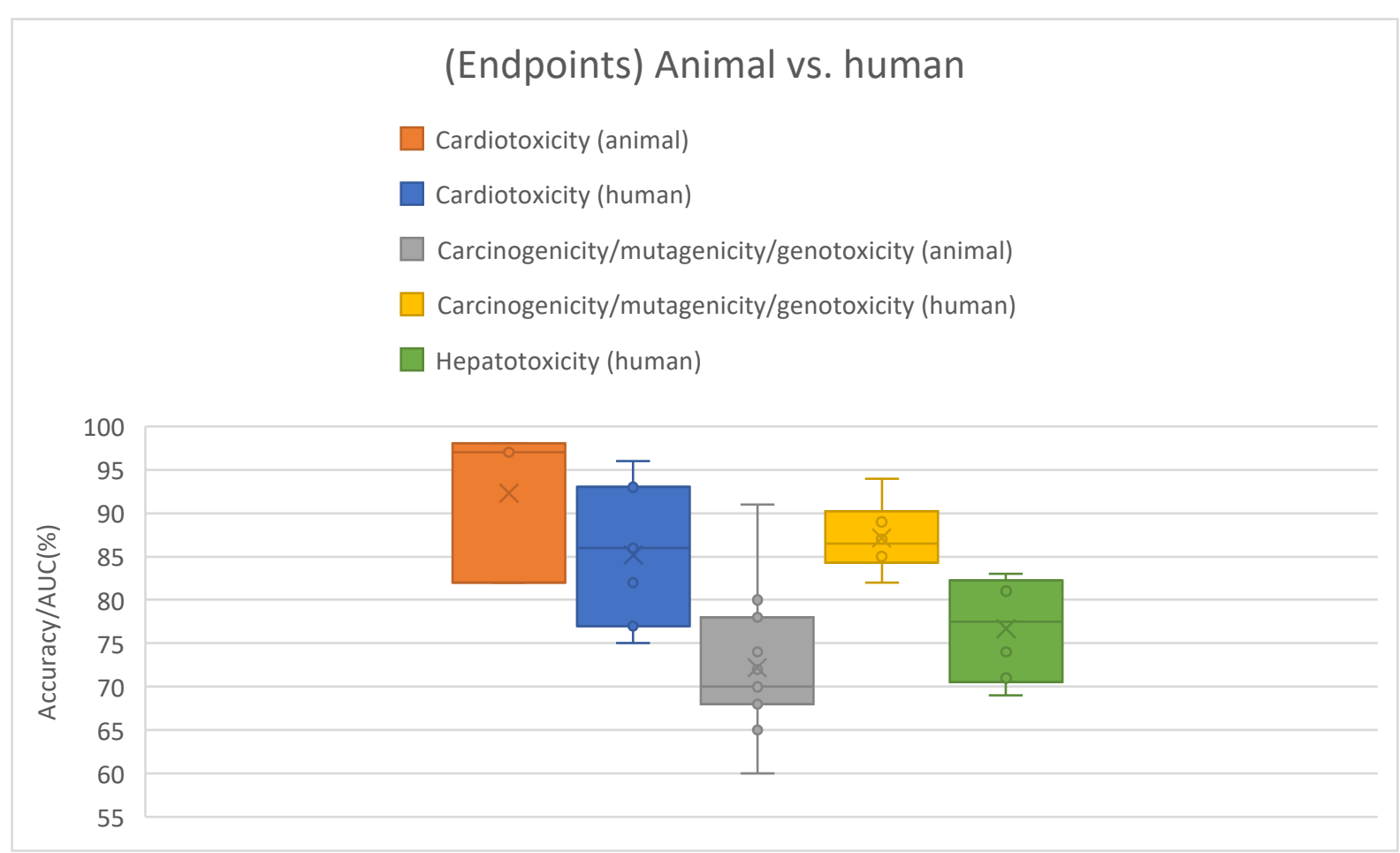

